\title{
Kiểm định sự hiện diện của bong bóng trên thị trường chứng khoán Việt Nam giai đoạn từ 2006 đến 2019
}

\section{Investigating the bubbles in Vietnam stock market during the period from 2006 to 2019}

\author{
Châu Đỗ Nhật Hậ ${ }^{1}$ Trần Thị Tuấn $\mathrm{Anh}^{1 *}$ \\ ${ }^{1}$ Trường Đại học Kinh tế Thành phố Hồ Chí Minh, Việt Nam \\ "Tác giả liên hệ, Email: anhttt@ ueh.edu.vn

THÔNG TIN TÓM TÁT \\ DOI: $10.46223 /$ HCMCOUJS. \\ econ.vi.15.1.250.2020 \\ Ngày nhận: 05/10/2019 \\ Ngày nhận lại: 04/11/2019 \\ Duyệt đăng: 15/11/2019 \\ Tù khóa: \\ bong bóng chứng khoán, \\ kiểm định GSADF, kiểm định \\ $\mathrm{SADF}$, thị trường chứng \\ khoán Việt Nam \\ Hiện tượng bong bóng xảy ra trên thị trường tài chính thường \\ dẫn đến một hệ lụy nghiêm trọng về nguy cơ sụp đổ thị trường \\ gây ra nhiều biến động cho nền kinh tế. Vì vậy, nhận diện sự tồn \\ tại bong bóng trên thị trường là một trong những chủ đề nghiên \\ cứu được quan tâm. Bài viết này sử dụng số liệu về giá đóng cửa \\ hàng ngày của chỉ số VN-Index và HNX-Index từ năm 2006 đến \\ cuối 2019 để kiểm định sự hiện diện của bong bong chứng khoán \\ trên cả hai thị trường chứng khoán Thành phố Hồ Chí Minh và Hà \\ Nội. Kết quả áp dụng kiểm định tính dừng phía phải bằng thống \\ kê (SADF - sup augmented Dickey-Fuller test) và kiểm định tính \\ dừng phía phải tổng quát (GSADF - generalized sup Augmented \\ Dickey-Fuller) trên dữ liệu của cả hai thị trường không những cho \\ thấy sự xuất hiện của bong bóng ở hai nơi, mà còn phát hiện được \\ thời gian xảy ra bong bóng trong từng giai đoạn 2006 - 2012 và \\ 2013 - 2019. Kết quả phân tích của bài viết cũng cho thấy sự tương \\ đồng trong hiện tượng bong bóng của hai chỉ số VN-Index và \\ HNX-Index, mặc dù có sự chênh lệch về thời gian, nhưng sự \\ chênh lệch này không đáng kể.
}

\begin{abstract}
The bubbles in the financial market often lead to the possibility of market collapse and harmful effects on the national economy. Therefore, identifying the existence of bubbles in the market is one of the most interesting research topics in finance. This article employs data of the daily closing prices of VN-Index and HNX-Index from 2006 to 2019 to investigate the presence of bubbles in both Ho Chi Minh and Hanoi Stock Exchanges. Analyzing both market data using the bubble-detection approaches of the sup augmented Dickey-Fuller test (SADF)
\end{abstract}


Keywords:

stock bubbles, generalized

Augmented Dickey-Fuller

test, Sup Augmented Dickey-

Fuller test, Vietnam stock

market and generalized sup Augmented Dickey-Fuller test (GSADF), the study not only demonstrates the presence of bubbles but also detects the time of bubbles occurring in periods 2006 - 2012 and 2013 - 2019. This reveals the similarity in the bubble phenomenon of the VN-Index and HNX-Index regardless of some minor differences of the time.

\section{Giới thiệu}

Thuật ngữ bong bóng kinh tế đã xuất hiện từ rất lâu trên thị trường tài chính. Bong bóng kinh tế thường được xem là nguyên nhân chủ yếu dẫn đến tình trạng khủng hoảng thị trường và dẫn đến nhiều hậu quả nặng nề cho nền kinh tế. Vì vậy, việc nhận ra sự hiện diện của bong bóng trên thị trường đóng vai trò rất quan trọng trong nghiên cứu kinh tế.

Reza (2010) giải thích về thuật ngữ bong bóng giá, thuật ngữ này thường được dùng để mô tả tình trạng thị trường liên tục ở trong trạng thái định giá quá cao một tài sản nào đó so với giá trị cơ sở hoặc giá trị thực của tài sản đó. Jones (2014) nhận định rằng bong bóng trên giá của một tài sản có thể phát sinh khi thị trường tiếp tục định giá tăng cho một tài sản vì tài sản này đã có một thời gian tăng giá trị trước đó. Khi giao dịch, các nhà đầu tư tin rằng vì giá trị của tài sản đã tăng lên trước đó, nên họ kỳ vọng giá sẽ tăng thêm trong một khoảng thời gian ngắn nữa và sẽ thu lợi nếu nắm giữ tài sản trong khoảng thời gian ngắn nữa; do đó nhà đầu tư quyết định mua tài sản với giá cao hơn so với giá trước đó. Điều này nói chung sẽ dẫn đến sự gia tăng hơn nữa giá tài sản khi nhu cầu về nó tăng lên. Sự tăng giá nối tiếp tăng giá làm hiện tượng bong bóng ngày càng trầm trọng và nguy cơ vỡ bong bóng cũng tăng lên cùng với nguy cơ sụp đổ của thị trường.

Với những lý thuyết thú vị về bong bóng thị trường nói chung và bong bóng chứng khoán nói riêng, bài viết này tiến hành kiểm định sự tồn tại của bong bóng trên thị trường chứng khoán Việt Nam từ năm 2006 đến 2019 với dữ liệu của chỉ số chứng khoán theo ngày trên thị trường. Phần còn lại của bài viết được tổ chức như sau: Mục 2 trình bày tóm tắt một số nghiên cứu về bong bóng trên thị trường trong nước và thế giới; Mục 3 đề cập phương pháp nghiên cứu; Mục 4 phân tích số liệu và thảo luận kết quả nghiên cứu; Mục 5 kết luận những kết quả nghiên cứu chính của đề tài.

\section{Tổng quan lý thuyết}

Hiện tượng bong bóng trên thị trường đã thu hút được nhiều sự quan tâm của các nghiên cứu. Rất nhiều các phương pháp đã được đề xuất nhằm kiểm định sự hiện diện của bong bóng trên thị trường. Một trong những phương pháp được sử dụng phổ biến đó là kiểm định Johansen (1988) để kiểm định đồng liên kết giữa giá và cổ tức của cổ phiếu. Nếu có mối liên hệ đồng liên kết xảy ra giữa giá và cổ tức thì việc tăng giá cổ phiếu gắn liền với việc tăng cổ tức và do đó, tăng giá trong trường hợp này không gây ra hiện tượng bong bóng.

Flood và Garber (1980) đã công bố mô hình kỳ vọng hợp lý (The Completed Rational Expectations Model) để thử nghiệm sự tồn tại đầu tiên của bong bóng. Mô hình kỳ vọng hợp lý trở thành cơ sở lý thuyết, nền tảng để đo lường bong bóng thị trường. Shiller (1981) đề xuất 
một thử nghiệm mới là kiểm định đường biên phương sai (Variance Bounds Tests) và tác giả tiến hành kiểm định trên dữ liệu mẫu của chỉ số giá S\&P từ năm 1871 đến 1979, đã đưa đến kết luận: "Bác bỏ giả thuyết null về không có bong bóng". Nhiều nghiên cứu khác sau đó cũng áp dụng phương pháp của Shiller nhưng cũng chỉ ra rằng kiểm định này sẽ cho kết quả không đáng tin cậy khi áp dụng trên mẫu nhỏ, Blanchard và Watson (1982) đã củng cố lập luận về việc giải thích bong bóng hợp lý là sự sai lệch của giá tài sản so với giá trị cơ bản; xuất phát từ thực tế là bong bóng đầu cơ không theo hành vi hợp lý, mặc dù hành vi hợp lý thực sự có ảnh hưởng đến các nguyên tắc cơ bản của thị trường và giá cả. Nhưng với sự can thiệp của các biến số bất hợp lý, không dễ để kiểm tra bong bóng trên thị trường chứng khoán.

West (1987) sử dụng đồng thời phương trình Euler và mô hình ARIMA của cổ tức, với dữ liệu giá cổ phiếu và cổ tức hàng năm của $S \& P 500$ trong giai đoạn 1871-1980 và chỉ số Dow Jones từ 1928 đến 1978 để kiểm định sự tồn tại của bong bóng chứng khoán trên thị trường Mỹ. Kết quả kiểm định cho thấy có bằng chứng thống kê mạnh mẽ về sự tồn tại của hiện tượng bong bóng trên thị trường.

Đến năm 1997, Wu (1997) đã đưa ra một lập luận mới - mô tả bong bóng là một biến không quan sát được. Dựa trên mô hình giá trị hiện tại (Present Value Model), ông đã tạo ra một mô hình tuyến tính dựa trên các thành phần bong bóng. Đặc biệt, $\mathrm{Wu}$ cho rằng cổ tức tuân theo quá trình tự hồi quy và ước tính bong bóng là một biến số không quan sát được với điều kiện không có chênh lệch giá khi sử dụng bộ lọc Kalman (Kalman Filter). Bộ lọc Kalman là một quy trình đệ quy để tính toán ước tính tối ưu của bong bóng tại mỗi khoảng thời gian, dựa trên mô hình cấu trúc kinh tế (The Structural Economic Model) và dữ liệu quan sát được. Ngoài ra, mô hình này thường cho thấy bong bóng tiêu cực. Kết quả kiểm định của ông ước tính được các thành phần bong bóng chiếm một tỷ lệ đáng kể trong S\&P 500 xuyên suốt những năm 1871 - 1992.

Herrera và Perry (2003) đã áp dụng kiểm định nghiệm đơn vị và đồng liên kết tiêu chuẩn để kiểm tra sự tồn tại của bong bóng hợp lý ở các nước Mỹ Latinh vào những năm 1980-2001. Phân tích của họ bao gồm hai bước. Trong bước đầu tiên, các tác giả kiểm định nghiệm đơn vị đối với tỷ lệ $\log$ của cổ tức và biến lợi nhuận. Các chuỗi có nghiệm đơn vị, thì chưa thể kết luận về sự tồn tại của bong bóng trong chuỗi giá của tài sản. Trong bước thứ hai, kiểm định đồng liên kết được sử dụng để kiểm tra mối quan hệ trong dài hạn giữa tỷ lệ log của cổ tức và tỷ suất sinh lợi cổ phiếu. Nếu một mối quan hệ trong dài hạn được kết luận thì đó là dấu hiệu không có bong bóng. Đối với hầu hết các quốc gia, Herrera và Perry nhận thấy rằng chuỗi cổ tức và lợi nhuận thực có nghiệm đơn vị; tiến hành kiểm định đồng liên kết thì kết quả cho thấy bác bỏ giả thuyết về mối quan hệ lâu dài giữa lợi nhuận thực và cổ tức ở tất cả các quốc gia.

Phillips, Wu và Yu (2011), viết tắt là PWY, đã đề xuất phương pháp kiểm định Dickey - Fuller phía phải (SADF - sup augmented Dickey-Fuller test) để kiểm định sự hiện diện của bong bóng chứng khoán. Kiểm định $\mathrm{SADF}$ của $\mathrm{PWY}$ dựa trên giả thuyết $\mathrm{H} 0$ về nghiệm đơn vị như kiểm định Dickey - Fuller truyền thống nhưng giả thuyết đối là giả thuyết phía phải. Nếu giả thuyết không trong kiểm định này bị bác bỏ, nghĩa là có bằng chứng thống kê cho hiện tượng bùng nổ trong chuỗi giá, cũng là bằng chứng cho sự hiện diện của bong bóng. Các kiểm định tính dừng phía phải SADF được thực hiện theo hình thức cửa sổ cuộn. Các kiểm định này được xem xét kiểm tra trong nghiên cứu của Homm và Breitung (2012) để phát hiện bong bóng chứng khoán trên thị trường. Sau quá trình mô phỏng và so sánh các tiêu chí đánh giá, các tác 
giả đã rút ra kết luận kiểm định SADF của Phillips và cộng sự (2011) là tối ưu nhất trong số các phương pháp áp dụng đến thời điểm này.

Kiểm định SADF hoạt động hiệu quả khi có một sự kiện bong bóng duy nhất, nhưng có bằng chứng cho thấy nhiều bong bóng có thể xuất hiện khi mẫu lớn $(\mathrm{Su}, \mathrm{Li}, \mathrm{Chang}, \&$ Lobonţ, 2017). Một ứng dụng thực nghiệm của phương pháp này được thực hiện trên dữ liệu giá và cổ tức thực của thị trường chứng khoán $\mathrm{S} \& \mathrm{P} 500$ trong giai đoạn lịch sử dài từ tháng 1 năm 1871 đến tháng 12 năm 2010. Cách tiếp cận mới xác định thành công các giai đoạn lịch sử nổi tiếng về sự xuất hiện và sụp đổ của bong bóng, xác định được các bong bóng chứng khoán thập niên 1990 nhưng hoàn toàn bỏ lỡ cuộc khủng hoảng nợ giai đoạn 2007 - 2008. Sự bỏ lỡ này cũng đã phần nào nói lên những hạn chế của kiểm định này. Nếu có hai bong bóng và thời gian bong bóng thứ hai ngắn hơn bong bóng thứ nhất, thì kiểm định SADF không thể ước tính được thời gian bắt đầu và kết thúc của bong bóng thứ hai. Ngoài ra, kiểm định có thể không thành công và không nhất quán trong việc tiết lộ sự tồn tại của bong bóng trong chuỗi thời gian dài và phân tích dữ liệu khi thị trường thay đổi nhanh chóng.

Khắc phục nhược điểm này, Phillips, Shi và Yu (2015), viết tắt là $\mathrm{PSY}$, đã phát triển thành công một phương pháp kiểm định mới là GSADF (Generalized sup ADF). Kiểm định GSADF cũng dựa trên kiểm định ADF phía phải, nhưng được áp dụng lặp đi lặp lại như kiểm định SADF theo dạng cửa sổ cuộn. Nó được thiết kế để tìm kiếm các hành vi dạng bùng nổ của thị trường trong các chuỗi mẫu xác định bởi cửa sổ cuộn. GSADF được xem là có độ linh hoạt của cửa sổ cuộn cao hơn $\mathrm{SADF}$, và là một phương pháp hiệu quả để khảo sát hành vi bùng nổ giá nhằm khẳng định sự hiện diện của bong bóng trên thị trường.

Năm 2015, Phillip áp dụng cả các kiểm định SADF, GSADF, cùng một số thuật toán khác cho tỷ lệ cổ tức của giá S\&P 500 từ tháng 1 năm 1871 đến tháng 12 năm 2010. Các kiểm định được áp dụng đều cho kết quả chứng minh về sự tồn tại bong bóng. Kết quả của kiểm định GSADF xác định hai giai đoạn bùng nổ: giai đoạn phục hồi của cơn hoảng loạn năm 1873 (tháng 10 năm 1879 đến tháng 4 năm 1880) và bong bóng Dot-com (tháng 7 năm 1997 đến tháng 8 năm 2001). Khi Phillip và cộng sự giới hạn thời gian bong bóng dài hơn mười hai tháng, kết quả kiểm định tìm thấy bằng chứng thống kê về 03 giai đoạn tồn tại bong bóng giá trên thị trường: bong bóng bùng nổ sau chiến tranh năm 1954, Black Monday vào tháng 10 năm 1987 và bong bóng Dot-com; trong khi kết quả kiểm định SADF của PWY chỉ xác định được bong bóng Dot-com trong số những trường hợp đó.

Từ một số kết quả trên, có thể thấy kiểm định GSADF là kiểm định hữu hiệu có thể phát hiện được sự hiện diện bong bóng trên thị trường và được áp dụng phổ biến trong các nghiên cứu bong bóng trên thị trường chứng khoán cũng như các thị trường tài sản tài chính khác. Với những ưu điểm này, bài viết sử dụng kiểm định $\mathrm{SADF}$ và GSADF để kiểm định sự tồn tại của bong bong giá trên thị trường Việt Nam giai đoạn 2006 đến 2009.

\section{Dữ liệu và phương pháp nghiên cứu}

\subsection{Dũ liệu}

Bài viết sử dụng dữ liệu là chỉ số giá đóng cửa của chỉ số VN-Index tại sàn giao dịch Thành phố Hồ Chí Minh và HNX-Index của thị trường chứng khoán Hà Nội trong thời gian từ tháng $1 / 2006$ đến tháng 6/2019. Dữ liệu được chia thành hai giai đoạn; giai đoạn đầu từ tháng 
1/2006 đến tháng 12/2012 và giai đoạn hai từ tháng 1/2013 đến tháng 6/2019. Mục đích của việc chia dữ liệu thành hai giai đoạn riêng biệt là để chứng minh sự chính xác của mô hình dựa vào thông tin đã được xác nhận: "Có sự tồn tại của bong bóng chứng khoán tại Việt Nam vào năm 2007" từ dữ liệu ở giai đoạn 1, đồng thời kiểm tra thị trường chứng khoán Việt Nam ở giai đoạn tiếp theo đến tháng 6/2019. Việc phân tích chính để tìm bằng chứng thống kê cho sự hiện diện của bong bóng được thực hiện trên chuỗi chỉ số VN-Index trong khi các kết quả kiểm định với số liệu HNX-Index được dùng để củng cố kết quả đạt được và đảm bảo tính ổn định trong kết quả nghiên cứu.

\subsection{Phưong pháp nghiên cúu}

Với tổng quan lý thuyết như trên, bài viết này áp dụng kiểm định giả thuyết SADF do Phillips và cộng sự (2011) đề xuất và kiểm định GSADF do Phillips và cộng sự (2015) đề xuất trên dữ liệu từ 2006 đến 2019 của chỉ số VN-Index và HNX-Index của sàn giao dịch Thành phố Hồ Chí Minh và Hà Nội. Việc bác bỏ giả thuyết không trong kiểm định này được xem như là bằng chứng thực nghiệm cho sự hiện diện của bong bóng giá tài sản tài chính. Giá trị tới hạn của các kiểm định này được tính toán thông qua phương pháp mô phỏng Monte Carlo và thông qua kết quả kiểm định cũng giúp xác định ngày bắt đầu và ngày kết thúc của bong bóng.

Cũng tương tự như trường hợp kiểm định tính dừng bằng Dickey-Fuller mở rộng, các kiểm định tính dừng phía phải SADF và GSADF xem xét phương trình hồi quy dạng sai phân

$$
\Delta y_{t}=\mu+\delta y_{t-1}+\sum_{i=1}^{p} \phi_{i} \Delta y_{t-1}+e_{t}
$$

Trong đó

$y_{t}$ : giá cổ phiếu tại thời điểm $t$

$\mu$ : hệ số chặn

$p$ : độ trễ tối đa

$\phi_{i}$ với $i=1 \ldots p$ là hệ số hồi quy ứng với các độ trễ khác nhau

$e_{t}$ : sai số

Khác với các kiểm định tính dừng thông thường, trong trường hợp cần kiểm tra sự hiện diện của bong bóng, giả thuyết $\mathrm{H}_{0}$ ứng với trường hợp chuỗi có nghiệm đơn vị được xem xét với giả thuyết đối phía; nghĩa là, cần thực hiện kiểm định:

$$
\begin{aligned}
& H_{0}: \delta=1 \\
& H_{1}: \delta>1
\end{aligned}
$$

Nếu giả thuyết $\mathrm{H}_{0}$ trong kiểm định này bị bác bỏ, nghĩa là có bằng chứng thống kê cho thấy hiện tượng bùng nổ trong chuỗi giá. Nếu hiện tượng này kéo dài trong một khoản thời gian nhất định thì bằng chứng về hiện tượng bong bóng càng được củng cố thêm. Khi thực hiện kiểm định, PWY giả sử khoảng thời gian mẫu là $[0,1]$. Ký hiệu $\delta_{r_{1}, r_{2}}$ và $A D F_{r_{1}, r_{2}}$ là hệ số ước lượng được theo phương trình (1) và kiểm định $\mathrm{ADF}$ tương ứng trong khung cửa sổ dữ liệu $\left[r_{1}, r_{2}\right]$. Nếu ký hiệu $\mathrm{r}_{\mathrm{w}}$ là kích thước cửa sổ, ta có $r_{w}=r_{2}-r_{1}$. Sự khác biệt giữa các kiểm định liên quan đến cách thiết lập $r_{1}$ và $r_{2}$. Thông thường khi tiến hành kiểm định nghiệm đơn vị, $r_{1}$ và $r_{2}$ được cố định cho các quan sát đầu tiên và cuối cùng, tương ứng là của toàn bộ mẫu, lúc này $r_{w}=r_{0}=1$. Tuy nhiên, trong kiểm định $\mathrm{SADF}$, được đề xuất bởi PWY, với điểm bắt đầu cố 
định và kích thước ban đầu của cửa sổ được chọn bởi người dùng; sau đó được mở rộng dần. Quy trình ước lượng như sau: Quan sát đầu tiên trong mẫu được đặt làm điểm bắt đầu của cửa sổ ước tính, $r_{1}$, tức là, $r_{1}=0$. Tiếp theo, điểm kết thúc của cửa sổ ước tính ban đầu, $r_{2}$, được đặt theo $r_{0}$, sao cho kích thước cửa sổ ban đầu là $r_{w}=r_{2}$. Cuối cùng, khi tăng kích thước cửa sổ thì $r_{2} \in\left[r_{0}, 1\right]$ trong mỗi lần quan sát. Mỗi ước tính mang lại một thống kê ADF được ký hiệu là $A D F_{r_{2}}$. Lưu ý rằng trong bước cuối cùng, ước tính sẽ dựa trên toàn bộ mẫu (tức là, $r_{2}=$ 1 và thống kê sẽ là $A D F_{1}$ ). Thống kê $\mathrm{SADF}$ được định nghĩa là giá trị nhỏ nhất (supremum) của chuỗi $A D F_{r_{2}}$ đối với các $r_{2} \in\left[r_{0}, 1\right]$ :

$$
\operatorname{SADF}\left(r_{0}\right)=\sup _{r_{2} \in\left[r_{0}, 1\right]}\left\{A D F_{r_{2}}\right\}
$$

Trong kiểm định GSADF được đề xuất bởi PSY, các bước thực hiện tương tự như kiểm định SADF của PWY nhưng tổng quát hơn hơn, thể hiện ở việc kiểm định GSADF cho phép các điểm bắt đầu, $r_{1}$, cũng được phép thay đổi trong phạm vi $\left[0, r_{2}-r_{0}\right]$. Thống kê GSADF được định nghĩa là:

$$
G \operatorname{SADF}\left(r_{0}\right)=\sup _{\substack{\left.r_{2} \in\left[r_{0}, 1\right] \\ r_{1} \in 0, r_{2}-r_{0}\right]}}\left\{\mathrm{ADF}_{r_{1}}^{r_{2}}\right\}
$$

PWY đề xuất so sánh từng yếu tố của chuỗi $A D F_{r_{2}}$ ước tính được với các giá trị tới hạn tương ứng với kiểm định phía phải của thống kê $\mathrm{ADF}$ chuẩn để xác định bong bóng bắt đầu tại thời điểm $T_{r_{2}}$. Điểm bắt đầu ước tính của bong bóng là quan sát thời gian đầu tiên, ký hiệu là $T_{r_{e}}$, trong đó $A D F_{r_{2}}$ vượt qua giá trị tới hạn tương ứng từ bên dưới, trong khi điểm kết thúc ước tính là quan sát theo thời gian đầu tiên sau $T_{r_{e}}$, được biểu thị bởi $T_{r_{f}}$ trong đó $A D F_{r_{2}}$ vượt qua tới hạn giá trị phía phải. Từ đó, các ước tính của thời kỳ bong bóng được xác định bởi:

$$
\begin{aligned}
& r_{e}=\inf _{r_{2} \in\left[r_{0}, 1\right]}\left\{r_{2}: A D F_{r_{2}}>c v_{r_{2}}^{\beta_{T}}\right\} \\
& r_{f}=\inf _{r_{2} \in\left[r_{e}, 1\right]}\left\{r_{2}: A D F_{r_{2}}>c v_{r_{2}}^{\beta_{T}}\right\}
\end{aligned}
$$

Trong đó: $c v_{r_{2}}^{\beta_{T}}$ là giá trị tới hạn $100\left(1-\beta_{T}\right) \%$ của thống kê ADF chuẩn dựa trên các quan sát $\left[\operatorname{Tr}_{2}\right]$.

Tương tự, các ước tính về thời kỳ bong bóng dựa trên GSADF được đưa ra bởi:

$$
\begin{aligned}
& r_{e}=\inf _{r_{2} \in\left[r_{0}, 1\right]}\left\{r_{2}: B S A D F_{r_{2}}\left(r_{0}\right)>c v_{r_{2}}^{\beta_{T r_{2}}}\right\} \\
& r_{f}=\inf _{r_{2} \in\left[r_{e}, 1\right]}\left\{r_{2}: B S A D F_{r_{2}}\left(r_{0}\right)>c v_{r_{2}}^{\beta_{T r_{2}}}\right\}
\end{aligned}
$$

Trong đó: $c v_{r_{2}}^{\beta_{T}}$ là giá trị tới hạn 100(1- $\left.\beta_{T}\right) \%$ của thống kê SADF dựa trên các quan sát $\left[\operatorname{Tr}_{2}\right]$. BSADF $\left(r_{0}\right)$ với $r_{2} \in\left[r_{0}, 1\right]$, là thống kê SADF trễ liên quan đến thống kê GSADF theo mối quan hệ sau: 


$$
\operatorname{GSADF}\left(r_{0}\right)=\sup _{r_{2} \in\left[r_{0}, 1\right]}\left\{B S A D F_{r_{2}}\left(r_{0}\right)\right\}
$$

\section{Kết quả nghiên cứu và thảo luận}

\subsection{Thống kê mô tả}

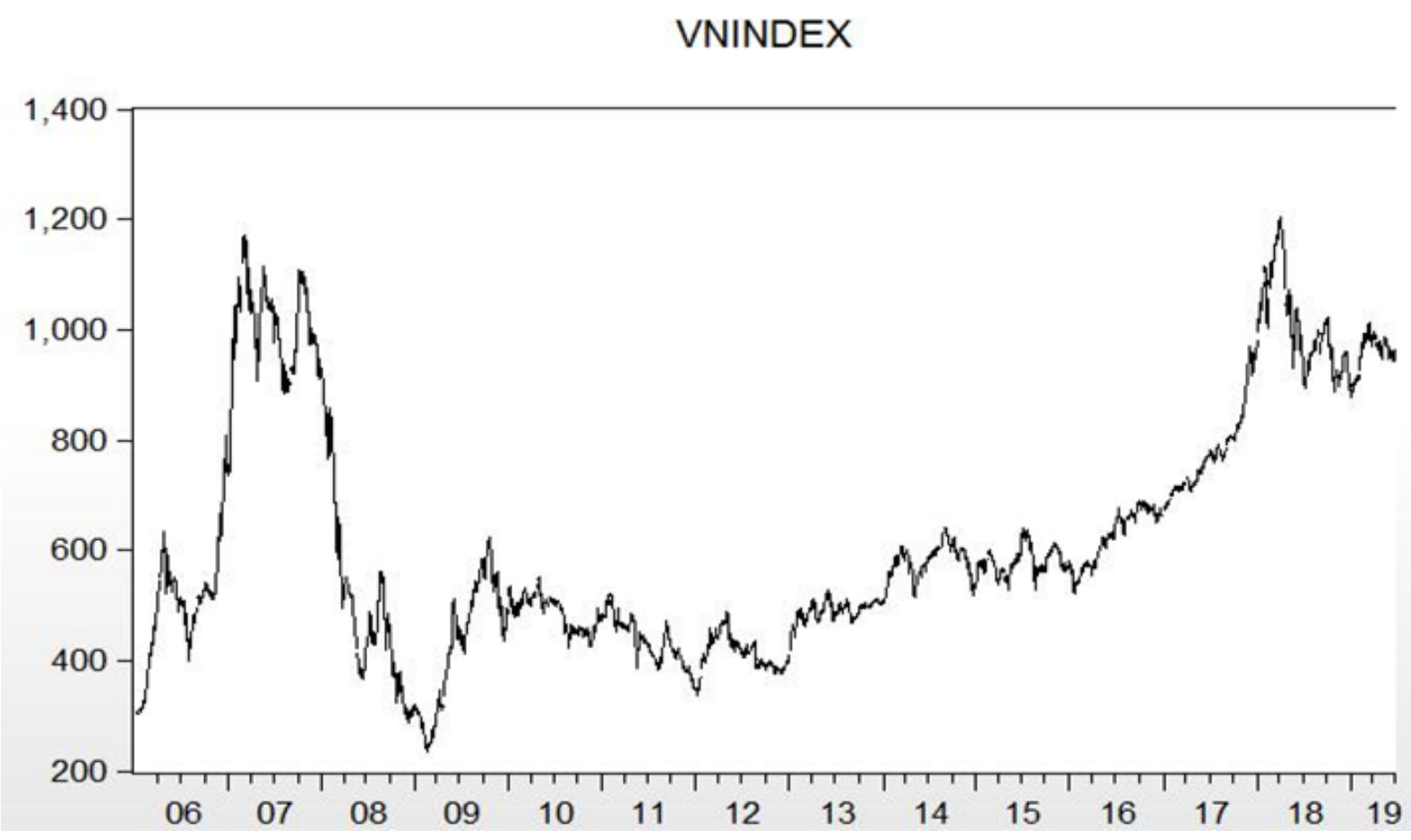

Hình 1. Biểu đồ chỉ số VN -Index từ 2006 đến 2019

\section{Bảng 1}

Mô tả chỉ số VN-Index và HNX-Index qua các năm

\begin{tabular}{lll}
\hline Năm & VN-Index & HNX-Index \\
\hline $\mathbf{2 0 0 6}$ & 505,91 & 186,55 \\
$\mathbf{2 0 0 7}$ & 1007,17 & 327,03 \\
$\mathbf{2 0 0 8}$ & 494,66 & 162,23 \\
$\mathbf{2 0 0 9}$ & 431,29 & 144,09 \\
$\mathbf{2 0 1 0}$ & 486,05 & 146,03 \\
$\mathbf{2 0 1 1}$ & 434,60 & 78,31 \\
$\mathbf{2 0 1 2}$ & 412,83 & 64,96 \\
$\mathbf{2 0 1 3}$ & 490,11 & 62,35
\end{tabular}




\begin{tabular}{llc}
\hline Năm & VN-Index & HNX-Index \\
\hline $\mathbf{2 0 1 4}$ & 579,95 & 82.16 \\
$\mathbf{2 0 1 5}$ & 579,88 & 82,41 \\
$\mathbf{2 0 1 6}$ & 625,91 & 81,40 \\
$\mathbf{2 0 1 7}$ & 780,17 & 98,13 \\
$\mathbf{2 0 1 8}$ & 1008,22 & 115,19 \\
$\mathbf{2 0 1 9}$ & 958,90 & 105,54 \\
\hline
\end{tabular}

Nguồn: Kết quả phân tích dữ liệu của nhóm nghiên cứu

Bảng 1 là kết quả thống kê mô tả giá trị trung bình của chỉ số VN-Index và HNX-Index từ năm 2006 đến năm 2019. Năm 2007 và năm 2018 là hai năm mà chỉ số VN-Index đạt giá trị vượt trội hơn cả, rõ ràng giá trị của VN-Index trong quá khứ đã tăng trưởng rất nhanh tính đến thời điểm hiện tại (6/2019) là 958,90. Vào thời điểm năm 2007 là năm đánh dấu sự xuất hiện của bong bóng trên thị trường chứng khoán Việt Nam, khi đó giá của VN-Index đạt mức đỉnh điểm là 1170,67 . Nhưng cho đến năm 2018 , cột mốc này đã bị phá vỡ, điều này dẫn đến một hoài nghi về bong bóng xuất hiện trên thị trường.

Cột 3 mô tả giá trị trung bình chỉ số HNX-Index từ giai đoạn 2006 đến 2019 của Sở giao dịch Chứng khoán Hà Nội. So với VN-Index đã xuất hiện từ năm 2000 thì HNX lại xuất phát trễ hơn 6 năm (2006), do đó thị trường chứng khoán ở Hà Nội vẫn chưa thể bắt kịp nhịp độ sôi động của thị trường Thành phố Hồ Chí Minh. Tuy nhiên, vào năm 2007, HNX-Index có sự tương đồng với VN-Index khi đây là năm mà giá của $\mathrm{HNX}$ chạm mốc cao nhất $(459,40)$ chỉ sau một năm thành lập.

\subsection{Kết quả nghiên cứu}

Trên thị truò̀ng chứng khoán Thành phố Hồ Chí Minh

\section{Bảng 2}

Kết quả SADF và GSADF của VN-Index giai đoạn 2006-2019

\begin{tabular}{|c|c|c|c|}
\hline Giai đoạn & Kết quả & Kiểm định SADF & Kiểm định GSADF \\
\hline \multirow{4}{*}{$\mathbf{2 0 0 6}-\mathbf{2 0 1 2}$} & $\begin{array}{c}\text { Thống kê kiểm } \\
\text { định }\end{array}$ & $3,0418^{* * *}$ & $4,7894 * * *$ \\
\cline { 2 - 4 } & $99 \%$ level & 2,0873 & 2,9979 \\
\cline { 2 - 4 } & $95 \%$ level & 1,5559 & 2,4834 \\
\cline { 2 - 4 } & $90 \%$ level & 1,3260 & 2,1722 \\
\hline \multirow{3}{*}{$\mathbf{2 0 1 3}-\mathbf{2 0 1 9}$} & $\begin{array}{c}\text { Thống kê kiểm } \\
\text { định }\end{array}$ & $3,5074 * * *$ & $4,9664 * * *$ \\
\cline { 2 - 4 } & $99 \%$ level & 2,0278 & 2,4877 \\
\cline { 2 - 4 } & $95 \%$ level & 1,5551 & 2,1747 \\
\hline
\end{tabular}




\begin{tabular}{|c|c|c|c|}
\hline Giai đoạn & Kết quả & Kiểm định SADF & Kiểm định GSADF \\
\hline & $90 \%$ level & 1,2738 & 2,0834 \\
\hline
\end{tabular}

Nguồn: Kết quả xử lý từ dữ liệu điều tra

Kết quả ở Bảng 2 cho thấy giá trị thống kê SADF và GSADF đều lớn hơn nhiều so với các giá trị tới hạn ở mức tin cậy $90 \%, 95 \%$ và 99 ; qua đó cho thấy bằng chứng thống kê về sự hiện diện của bong bóng chứng khoán trên sàn giao dịch TP.HCM trong cả hai giai đoạn 2006 - 2012 và 2013 - 2019.

Trong Hình 2, đường trên cùng là giá chứng khoán VN-Index thực tế theo ngày vào giai đoạn 2006 - 2012, trong khi đường ở dưới biểu thị cho kiểm định SADF và đường giữa là giá trị tới hạn $95 \%$. Ngày bắt đầu của bong bóng có thể được kiểm tra dễ dàng bằng mắt, khi đường biểu thị cho kiểm định SADF vượt qua đường giá trị tới hạn $95 \%$ từ bên dưới, tương ứng với sự tăng giá của VN-Index. Ngược lại, thời điểm kết thúc của bong bóng được xác định, khi đường biểu thị cho kiểm định $\mathrm{SADF}$ vượt qua đường giá trị tới hạn $95 \%$ từ phía trên. Trong Hình 2, kiểm định SADF đã giúp phát hiện một đợt bong bóng của VN-Index vào giai đoạn 2006 - 2012, cụ thể là cuối năm 2006 cho đến tháng 5 năm 2007.

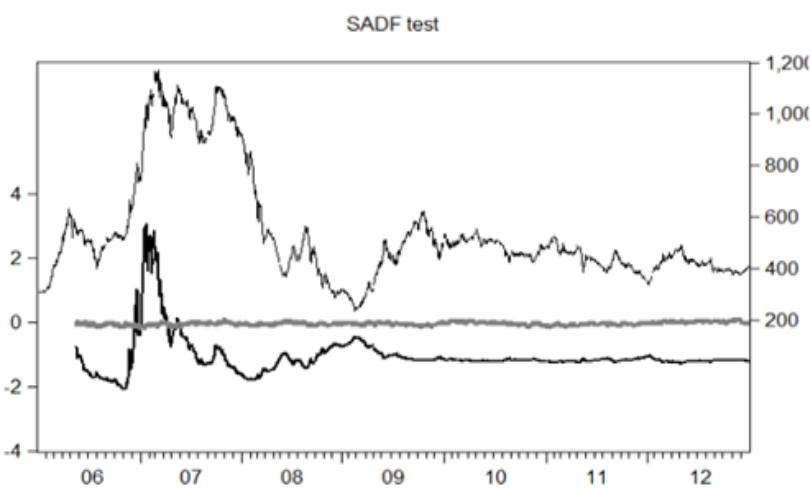

(a) SADF - Giai đoạn 2006 - 2012

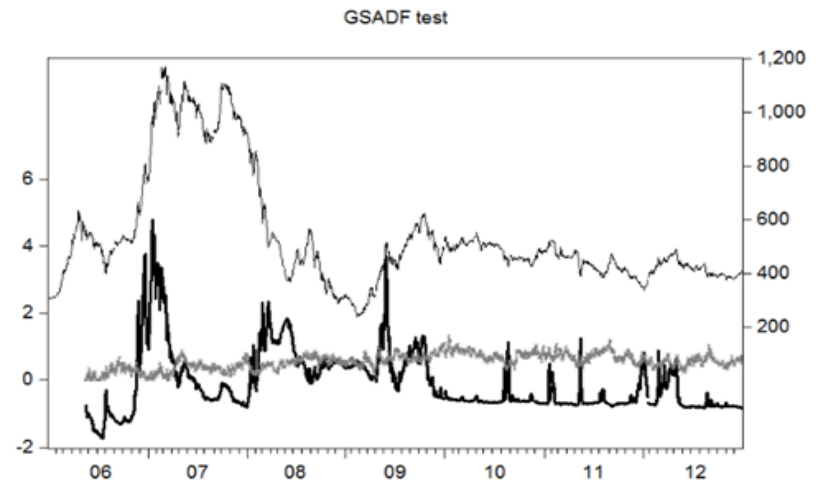

(c) GSADF - Giai đoạn 2006 - 2012

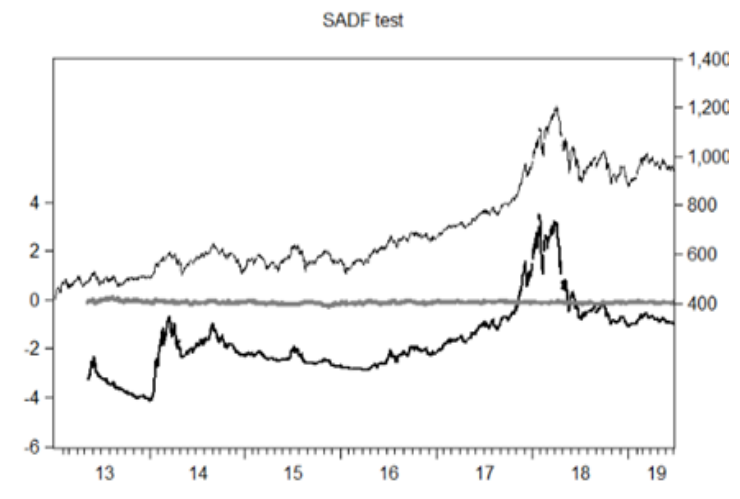

(b) SADF - Giai đoạn 2013 - 2019

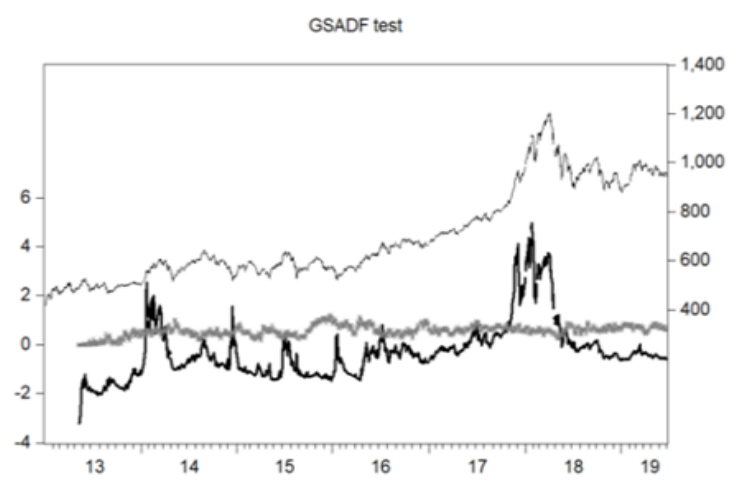

(d) GSADF - Giai đoạn 2013 - 2019

Hình 2. Biểu đồ kết quả SADF và GSADF của VN - Index giai đoạn 2006-2019

Trên thị trưòng chúng khoán Hà Nội

Tương tự VN-Index, đề tài đã thực hiện kiểm định SADF và GSADF để kiểm tra sự tồn 
tại của bong bóng cho chỉ số $\mathrm{HNX}$, với kích thước cửa sổ tối thiểu 91 làm kích thước cửa sổ ban đầu (initial window size), các giá trị tới hạn được lấy thông qua 100 lần lặp mô phỏng MC. Dữ liệu mẫu giai đoạn 2006 đến 2012 gồm 1697 quan sát; và dữ liệu mẫu giai đoạn 2013-1019 gồm 1615 quan sát.

\section{Bảng 3}

Kết quả kiểm định SADF và GSADF của HNX-Index

\begin{tabular}{|c|c|c|c|}
\hline Giai đoạn & & Kiểm định SADF & Kiểm định GSADF \\
\hline \multirow{4}{*}{2006 - 2012 } & Thống kê kiểm định & $2,3762 * * *$ & $4,3964 * * *$ \\
\cline { 2 - 4 } & $99 \%$ level & 2,3103 & 2,9883 \\
\cline { 2 - 4 } & $95 \%$ level & 1,6367 & 2,5720 \\
\cline { 2 - 4 } & $90 \%$ level & 1,3425 & 2,2607 \\
\hline \multirow{3}{*}{$\mathbf{2 0 1 3 - 2 0 1 9}$} & Thống kê kiểm định & $3,7617 * * *$ & $5,6784 * *$ \\
\cline { 2 - 4 } & $99 \%$ level & 2,0620 & 2,9553 \\
\cline { 2 - 4 } & $95 \%$ level & 1,5465 & 2,4578 \\
\cline { 2 - 4 } & $90 \%$ level & 1,2977 & 2,2607 \\
\hline
\end{tabular}

Nguồn: Kết quả xử lý từ dữ liệu điều tra

Ở Bảng 3 , kết quả kiểm định SADF và GSADF từ dữ liệu mẫu trong cả hai giai đoạn 2006 - 2012 và 2013 - 2019 đều lớn hơn giá trị tới hạn có mức ý nghĩa 10\%, 5\% và 1\%; vì vậy bác bỏ giả thuyết không có bong bóng. Đây là bằng chứng cho sự hiện diện của bong bóng trên sàn giao dịch Hà Nội trong cả hai giai đoạn dữ liệu được phân tích. Hình 3 thể hiện kết quả kiểm định bằng biểu đồ cho thấy rõ sự tồn tại của một bong bóng trong giai đoạn 2006 đến 2012, cụ thể là tháng $1 / 2007$ đến tháng 3/2007; và một bong bóng khác trong thời gian từ 6/2017 đến $5 / 2018$.

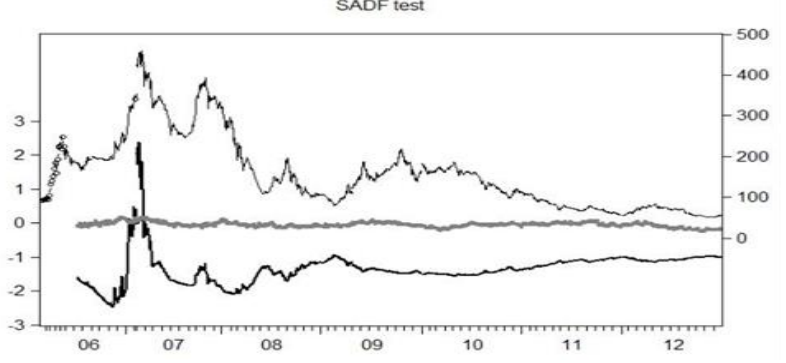

(a) SADF - Giai đoạn $2006-2012$

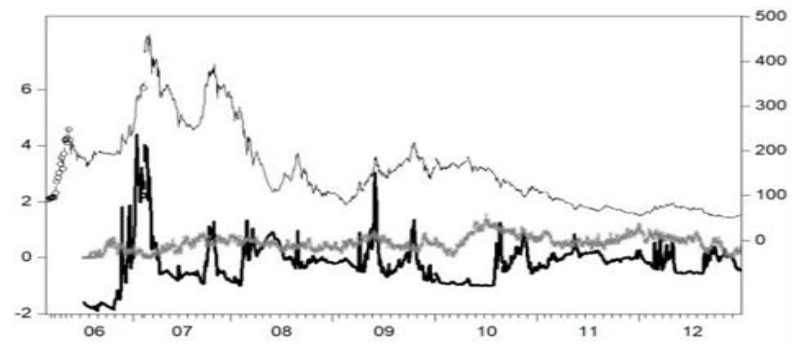

(c) GSADF - Giai đoạn 2006 - 2012

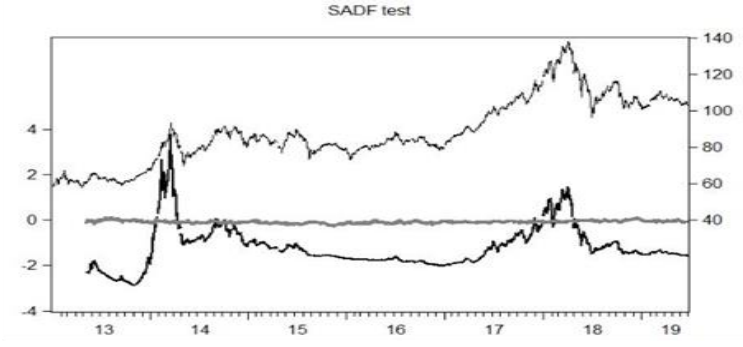

(b) SADF - Giai đoạn 2013 - 2019

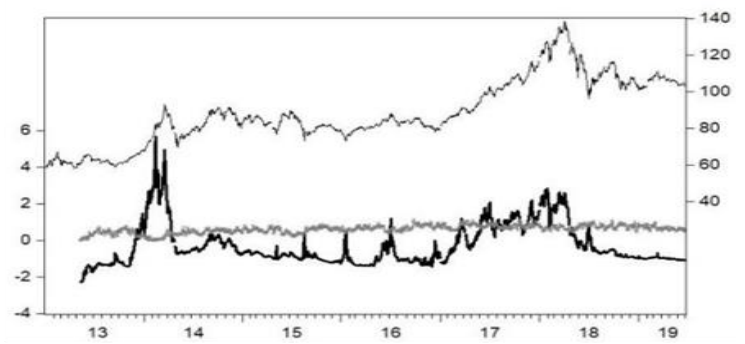

(d) GSADF - Giai đoạn 2013 - 2019

Hình 3. Biểu đồ kết quả SADF và GSADF của VN -Index giai đoạn 2006-2019 


\section{Kết luận}

Bài viết sử dụng số liệu về giá đóng cửa hàng ngày của chỉ số VN-Index và HNX-Index ở hai giai đoạn; giai đoạn thứ nhất là từ năm 2006 đến cuối 2012, giai đoạn tiếp theo từ 2013 đến cuối tháng 6 năm 2019. Hai chỉ số này đại diện cho hai sàn giao dịch chứng khoán lớn nhất ở Việt Nam; VN-Index đại diện cho Sàn giao dịch HOSE (miền Nam), thì HNX-Index đại diện cho sàn giao dịch chứng khoán Hà Nội (miền Bắc). Khi áp dụng kiểm định SADF và kiểm định GSADF cho hai tập dữ liệu của cả hai thị trường không những cho thấy sự xuất hiện của bong bóng "tích cực" ở hai chỉ số, mà còn phát hiện được thời gian xảy ra bong bóng trong giai đoạn khủng hoảng (2007 đến 2008). Kết quả phân tích của bài viết cũng cho thấy sự tương đồng trong hiện tượng bong bóng của hai chỉ số VN-Index và HNX-Index, mặc dù có sự chênh lệch về thời gian, nhưng vấn đề này không đáng kể.

Kể từ khi thành lập cho đến nay, thị trường chứng khoán Việt Nam tuy gặp nhiều biến động, thử thách và khó khăn nhưng vẫn ngày một phát triển. Những nhà đầu tư chứng khoán thận trọng khi đầu tư và rút ra bài học cho bản thân từ những biến cố bong bóng dẫn đến thị trường sụp đổ trong lịch sử của thị trường chứng khoán thế giới nói chung và Việt Nam nói riêng. Hai kiểm định này chỉ có thể xác định được bong bóng bằng dữ liệu có sẵn trong quá khứ, qua đó cung cấp một công cụ kiểm định mang tính chất dự báo, giúp cảnh báo cho nhà đầu tư về sự xuất hiện của bong bóng ở tương lai.

\section{Tài liệu tham khảo}

Abhyankar, A., Copeland, L. S., \& Wong, W. (1997). Uncovering nonlinear structure in realtime stock-market indexes: The S\&P 500, the DAX, the Nikkei 225, and the FTSE-100. Journal of Business \& Economic Statistics, 15(1), 1-14.

Blanchard, O. J., \& Watson, M. W. (1982). Bubbles, rational expectations and financial markets. Cambridge, MA: National Bureau of Economic Research.

Flood, R. P., \& Garber, P. M. (1980). Market fundamentals versus price-level bubbles: The first tests. Journal of Political Economy, 88(4), 745-770.

Herrera, S., \& Perry, G. E. (2003). Tropical bubbles: Asset prices in Latin America, 1980-2001. In W.C. Hunter, G. G. Kaufman \& M. Pomerleano (Eds.), Asset price bubbles: The implications for monetary, regulatory, and international policies (pp. 127-162). Cambridge, MA: MIT Press.

Homm, U., \& Breitung, J. (2012). Testing for speculative bubbles in stock markets: A comparison of alternative methods. Journal of Financial Econometrics, 10(1), 198-231.

Johansen, S. (1988). Statistical analysis of cointegration vectors. Journal of Economic Dynamics and Control, 12(2/3), 231-254.

Jones, B. (2014). Identifying speculative bubbles: A two-pillar surveillance framework. (Working Paper No. 14/208). doi:10.5089/9781498332071.001

Phillips, P. C. B., Shi, S. P., \& Yu, J. (2015). Testing for multiple bubbles: Historical episodes of exuberance and collapse in the S\&P 500. International Economic Review, 56(4), 10431078. 
Phillips, P. C. B., Wu, Y., \& Yu, J. (2011). Explosive behavior in the 1990s Nasdaq: When did exuberance escalate asset values? International Economic Review, 52(1), 201-226.

Phillips, P. C. B., \& Yu, J. (2009). Limit theory for dating the origination and collapse of mildly explosive periods in time series data. Paper presented at Singapore Management University, Singapore.

Reza, B. S.-M. (2010). Literatures about asset price bubbles and monetary policies. Proceedings of International Conference on Applied Economics, 695-703.

Shiller, R. J. (1981). Do stock prices move too much to be justified by subsequent changes in dividends? American Economic Review, 71(3), 421-436.

Su, C. W., Li, Z. Z., Chang, H. L., \& Lobonţ, O. R. (2017). When will occur the crude oil bubbles? Energy Policy, 102, 1-6.

West, K. D. (1987). A specification test for speculative bubbles. The Quarterly Journal of Economics, 102(3), 553-580.

Wu, Y. (1997), Rational bubbles in the stock market: Accounting for the U.S. stock-price olatility. Economic Inquiry, 35(2), 309-319. 